\title{
A screening for essential cell growth-related genes involved in arsenite toxicity in Saccharomyces cerevisiae
}

\author{
Tsutomu Takahashi, Shohei Satake, Ken-ichiro Hirose, Gi-Wook Hwang \\ and Akira Naganuma \\ Laboratory of Molecular and Biochemical Toxicology, Graduate School of Pharmaceutical Sciences, \\ Tohoku University, Sendai, Miyagi 980-8578, Japan
}

(Received September 10, 2011; Accepted October 17, 2011)

\begin{abstract}
Genes that are essential for growth in yeast were screened to identify those involved in arsenite sensitivity. We found that the knockdown of YPT1, ERG8, or RKI1 enhanced arsenite sensitivity in yeast.
\end{abstract}

Key words: Arsenite, Yeast, YPT1, ERG8, RKI1

\section{INTRODUCTION}

Arsenic is a trace element ubiquitously present in the environment. The excessive intake of arsenic compounds causes health problems, such as skin pigmentation and cancers (Alam et al., 2002; Ratnaike, 2003). The molecular mechanisms underlying the manifestation of arsenic toxicity include the inhibition of enzyme activity through reactions with the thiol group of various enzymes (Petrick et al., 2001; Shi et al., 2004), increased production of reactive oxygen species (Jomova et al., 2011), and apoptosis induction through the activation of a signal transduction mechanism (Kumagai and Sumi, 2007; Sumi et al., 2010). However, the detailed mechanisms that mediate arsenic toxicity remain unclear.

The budding yeast, Saccharomyces cerevisiae, has been used as a eukaryotic model in various molecular biological studies (Breitkreutz et al., 2010; Tarassov et al., 2008). A gene deletion yeast library has been used for the screening of genes involved in sensitivity to various chemicals, such as arsenic compounds (Thorsen et al., 2009; Aouida et al., 2004; Zhou et al., 2009). However, genes essential for survival have barely been examined. Thus, we herein explored genes that influenced sensitivity to arsenite, a trivalent inorganic arsenic compound that is among those essential for yeast survival.

\section{MATERIALS AND METHODS}

\section{Yeast strains and media}

The Decreased Abundance by mRNA Perturbation (DAmP) yeast library (Open Biosystems, Huntsville, AL, USA) shows the decreased expression of each essential gene through mRNA perturbation (Breslow et al., 2008). This library contains essential gene hypomorphic alleles whereby the 3' untranslated region (UTR) of each essential gene in the collection has been disrupted with an antibiotic resistance cassette. The DAmP yeast strains and the

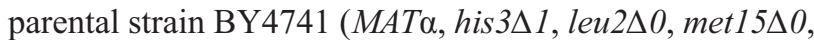
ura $3 \Delta 0$ ) were grown in yeast extract peptone-adeninedextrose (YAPD) medium or in synthetic dextrose (SD) medium.

\section{Screening for arsenite-hypersensitive yeast strains through the knockdown of essential genes}

The DAmP yeast strains with knockdown of essential genes were cultured in YPAD medium $(120 \mu 1)$ in 96-well plates for $48 \mathrm{hr}$ at $30^{\circ} \mathrm{C}$. Each culture was diluted $1 / 40$ with SD medium, and the aliquots $(5 \mu \mathrm{l})$ were transferred to fresh SD medium $(195 \mu \mathrm{l})$ containing $1.5 \mathrm{mM}$ sodium arsenite in 96-well plates for $48 \mathrm{hr}$. Under these culture conditions, the parental strain BY4741 exhibited increased growth. After a $48 \mathrm{hr}$ incubation, deletion mutants not exhibiting increased growth were identified as candidates for designation as arsenite-hypersensitive mutants.

Correspondence: Akira Naganuma (E-mail: naganuma@m.tohoku.ac.jp) 


\section{Measurement of the sensitivity of yeast cells to arsenite}

For spot assays using agar-solidified SD medium, a suspension of yeast cells was spotted onto a plate of agarsolidified SD medium with or without sodium arsenite. Plates were photographed after incubation for $48 \mathrm{hr}$ at $30^{\circ} \mathrm{C}$.

\section{Confirmation of knockdown efficiency using quantitative real-time PCR}

Isolation of total RNA from yeast cells was performed as described by Furuchi et al. (Furuchi et al., 2004). cDNAs were prepared from total RNA using the PrimeScript $^{\mathrm{TM}}$ RT reagent kit (Takara, Shiga, Japan) (Hwang et al., 2011). We performed quantitative real-time PCR using the Thermal Cycler Dice Real Time system (Takara) and SYBR Premix EX Taq (Takara). The oligonucleotide sequences of the primers used for quantitative PCR were as follows: sense, 5' - CAAGCGTGTCGTGGAATATG-3', and antisense, 5' - CCTTGATTTGTCTAGCCATGG -3' for YPT1; sense, 5'- ATCTGGTGCCGATATCGAAC -3', and antisense, 5' - GCGTCATAACCACCAGCACC -3' for $E R G 8$; sense, 5' - GAGGCAAGGTGTTCCCATTG-3', and antisense, 5'- GCTTTAGCAGAACCTCCTTGTC-3' for RKI1; and sense, 5' - TTGGATTCCGGTGATGGTGTTACT -3', and antisense, 5'- TGAAGAAGATTGAGCAGCGGTTTG -3' for $A C T 1$. The mRNA levels were normalized to those of $A C T 1$.

\section{RESULTS AND DISCUSSION}

Essential genes for yeast survival were screened to identify those involved in arsenite sensitivity. A yeast strain library, in which 878 of about 1,000 genes essential for the survival of budding yeast were suppressed through 3' UTR disruption, was used for screening. In this library, a kanamycin resistance gene is inserted between the termination codon and terminator of each gene in the genome to destabilize mRNA and suppress gene expression (Breslow et al., 2008). The knockdown yeast strains, in which the expression of each gene was suppressed, were cultured with arsenite at a concentration that allowed the growth of the parent strain BY4741. Then, arsenite-sensitive strains, which showed no growth even at $48 \mathrm{hr}$, were obtained. The screening demonstrated that yeasts with YPT1, ERG8, or RKI1 knockdown were more sensitive than wild-type yeast (Fig. 1A). Gene expression levels in the knockdown yeast strains were examined by realtime PCR. Gene expression levels were suppressed by about 70,60 , and $80 \%$ in YPT1, ERG8, and RKI1 knockdown yeast strains, respectively (Fig. 1B). The YPT1
A

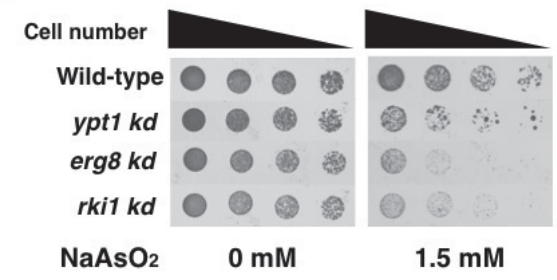

B
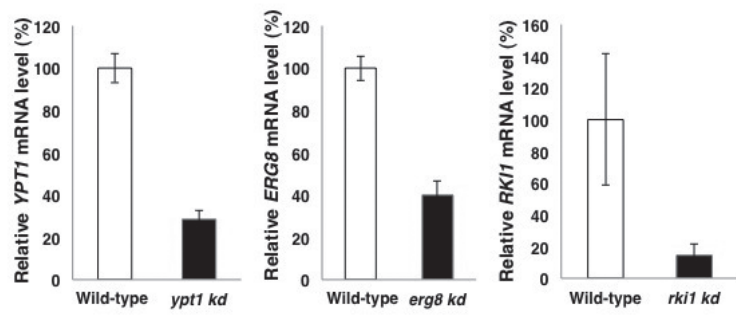

Fig. 1. Effects of knockdown of YPT1, ERG8, or RKI1 on the sensitivity of yeast cells to arsenite. (A) Yeast cells with knockdown $(\mathrm{kd})$ of $Y P T 1, E R G 8$, or $R K I 1$ were serially diluted in liquid-SD medium and spotted on agar-solidified SD medium prepared with and without $1.5 \mathrm{mM}$ arsenite. The plates were photographed after incubation for two days at $30^{\circ} \mathrm{C}$. The data are representative of three independent experiments. (B) The mRNA levels for each of the three genes in the yeast cells were analyzed using quantitative PCR. For comparison of mRNA expression, the mRNA level for each gene was normalized to that of $A C T 1$.

gene encodes Rab family GTPase in the intracellular vesicular transport pathway (Benito-Moreno et al., 1994; Miaczynska et al., 2001). The ERG8 gene encodes phosphomevalonate kinase in the mevalonate pathway (Tsay and Robinson, 1991). The RKI1 gene encodes ribose-5phosphate ketol-isomerase in the pentose phosphate pathway (Kondo et al., 2004). None of the genes identified by this screening have been examined regarding their relationships with arsenic toxicity. Thus, examining the relationships between these genes and arsenite toxicity may reveal unknown mechanisms of arsenic toxicity.

\section{REFERENCES}

Alam, M.G., Allinson, G., Stagnitti, F., Tanaka, A. and Westbrooke, M. (2002): Arsenic contamination in Bangladesh groundwater: a major environmental and social disaster. Int. J. Environ. Health Res., 12, 235-253.

Aouida, M., Page, N., Leduc, A., Peter, M. and Ramotar, D. (2004): A genome-wide screen in Saccharomyces cerevisiae reveals altered transport as a mechanism of resistance to the anticancer drug bleomycin. Cancer Res., 64, 1102-1109.

Benito-Moreno, R.M., Miaczynska, M., Bauer, B.E., Schweyen, R.J. and Ragnini, A. (1994): Mrs6p, the yeast homologue of the 
Identification of three novel arsenite-resistant genes in budding yeast

mammalian choroideraemia protein: immunological evidence for its function as the Ypt1p Rab escort protein. Curr. Genet., 27, 23-25.

Breitkreutz, A., Choi, H., Sharom, J.R., Boucher, L., Neduva, V., Larsen, B., Lin, Z.Y., Breitkreutz, B.J., Stark, C., Liu, G., Ahn, J., Dewar-Darch, D., Reguly, T., Tang, X., Almeida, R., Qin, Z.S., Pawson, T., Gingras, A.C., Nesvizhskii, A.I. and Tyers, M. (2010): A global protein kinase and phosphatase interaction network in yeast. Science, 328, 1043-1046.

Breslow, D.K., Cameron, D.M., Collins, S.R., Schuldiner, M., Stewart-Ornstein, J., Newman, H.W., Braun, S., Madhani, H.D., Krogan, N.J. and Weissman, J.S. (2008): A comprehensive strategy enabling high-resolution functional analysis of the yeast genome. Nat. Methods, 5, 711-718.

Furuchi, T., Takahashi, T., Tanaka, S., Nitta, K. and Naganuma, A. (2004): Functions of yeast helicase Ss12p that are essential for viability are also involved in protection from the toxicity of adriamycin. Nucleic Acids Res., 32, 2578-2585.

Hwang, G.W., Lee, J.Y., Ryoke, K., Matsuyama, F., Kim, J.M., Takahashi, T. and Naganuma, A. (2011): Gene expression profiling using DNA microarray analysis of the cerebellum of mice treated with methylmercury. J. Toxicol. Sci., 36, 389-391.

Jomova, K., Jenisova, Z., Feszterova, M., Baros, S., Liska, J., Hudecova, D., Rhodes, C.J. and Valko, M. (2011): Arsenic: toxicity, oxidative stress and human disease. J. Appl. Toxicol., 31, 95-107.

Kondo, H., Nakamura, Y., Dong, Y.X., Nikawa, J. and Sueda, S. (2004): Pyridoxine biosynthesis in yeast: participation of ribose 5-phosphate ketol-isomerase. Biochem. J., 379, 65-70.

Kumagai, Y. and Sumi, D. (2007): Arsenic: signal transduction, transcription factor, and biotransformation involved in cellular response and toxicity. Annu. Rev. Pharmacol. Toxicol., 47, 243262.
Miaczynska, M., Wagner, W., Bauer, B.E., Schweyen, R.J. and Ragnini, A. (2001): Ypt protein prenylation depends on the interplay among levels of Rab escort protein and geranylgeranyl diphosphate in yeast cells. Yeast, 18, 697-709.

Petrick, J.S., Jagadish, B., Mash, E.A. and Aposhian, H.V. (2001): Monomethylarsonous acid (MMA(III)) and arsenite: LD(50) in hamsters and in vitro inhibition of pyruvate dehydrogenase. Chem. Res. Toxicol., 14, 651-656.

Ratnaike, R.N. (2003): Acute and chronic arsenic toxicity. Postgrad. Med. J., 79, 391-396.

Shi, H., Shi, X. and Liu, K.J. (2004): Oxidative mechanism of arsenic toxicity and carcinogenesis. Mol. Cell. Biochem., 255, 67-78.

Sumi, D., Shinkai, Y. and Kumagai, Y. (2010): Signal transduction pathways and transcription factors triggered by arsenic trioxide in leukemia cells. Toxicol. Appl. Pharmacol., 244, 385-392.

Tarassov, K., Messier, V., Landry, C.R., Radinovic, S., Serna, Molina, M.M., Shames I., Malitskaya, Y., Vogel, J., Bussey, H. and Michnick, S.W. (2008): An in vivo map of the yeast protein interactome. Science, 320, 1465-1470.

Thorsen, M., Perrone, G.G., Kristiansson, E., Traini, M., Ye, T., Dawes, I.W., Nerman, O. and Tamás, M.J. (2009): Genetic basis of arsenite and cadmium tolerance in Saccharomyces cerevisiae. BMC Genomics, 10, 105.

Tsay, Y.H. and Robinson, G.W. (1991): Cloning and characterization of ERG8, an essential gene of Saccharomyces cerevisiae that encodes phosphomevalonate kinase. Mol. Cell. Biol., 11, 620-631.

Zhou, X., Arita, A., Ellen, T.P., Liu, X., Bai, J., Rooney, J.P., Kurtz, A.D., Klein, C.B., Dai, W., Begley, T.J. and Costa, M. (2009): A genome-wide screen in Saccharomyces cerevisiae reveals pathways affected by arsenic toxicity. Genomics, 94, 294-307. 